involves treating urban backyard chickens with ivermectin (IVM), a widely used antiparasitic and mosquitocial drug. The goal was to reduce vector mosquito populations and West Nile virus (WNV) transmission. METHODS/STUDY POPULATION: We placed eight flocksfour treated and four untreated control — of six Lohmann brown chickens (16 month-old) each in backyard coops across Davis, CA and administered IVM in feed daily at treated coops (200 $\mathrm{mg}$ IVM $/ \mathrm{kg}$ feed) for eleven weeks. We monitored entomological indices weekly (i.e. mosquito abundance, WNV infection prevalence, and parity rate) in Culex mosquito populations near $(10 \mathrm{~m})$ and far $(150 \mathrm{~m})$ from each coop location for the peak WNV transmission season (Jul-Sep 2019). We also monitored serum IVM levels in treated chickens and tested for WNV antibodies in all chickens throughout the study. RESULTS/ANTICIPATED RESULTS: Since IVM impacts only mosquitoes that live long enough to take a bloodmeal from a treated chicken, we do not expect to find a marked difference in adult Culex abundance between the two treatment arms, but we expect to find a reduction in WNV infection prevalence and a shift in female mosquito age structure towards younger, uninfected individuals at treated coops. We also anticipate seroconversions in treated chickens to occur at lower rates versus untreated control chickens indicating a reduction in WNV transmission intensity at treated coops. We observed no negative health outcomes from the long-term ingestion of IVM by study chickens. A pathological investigation is underway to compare histological findings between treated and untreated chickens. DISCUSSION/ SIGNIFICANCE OF IMPACT:IVM provides the potential for targeted mosquito control. Reduced WNV transmission dynamics here is a stepping stone to a commercial WNV control strategy; IVM-treated feed for wild birds for homeowners' use to combat WNV transmission in their neighborhoods.

\section{Association Between Injury Intent and Emergency Department and Hospital Charges for Pediatric Firearm Injuries in the United States*}

Diana Marie Bongiorno ${ }^{1}$, Gia M. Badolato ${ }^{2}$, Meleah D. Boyle ${ }^{2}$, Jon S. Vernick $^{3}$, Joseph F. Levy ${ }^{3}$, and Monika K. Goyal ${ }^{2}$

${ }^{1}$ Johns Hopkins University School of Medicine; ${ }^{2}$ Children's National Health System; ${ }^{3}$ Johns Hopkins University Bloomberg School of Public Health

OBJECTIVES/GOALS: In 2016, more than 3,100 children died, and an estimated 17,000 children had non-fatal injuries, from firearms in the United States. In this study, we used hospital charges as a proxy for medical resource utilization, and compared differences in charges by intent of firearm injury among children. METHODS/STUDY POPULATION: In this cross-sectional study of the 2016 Nationwide Emergency Department Sample, we identified firearm injury cases among children aged 19 years or younger using ICD-10-CM external cause of morbidity codes. Injury intent was characterized as unintentional, assault, selfinflicted, undetermined, or due to legal intervention. We included patients treated and released from the emergency department (ED) or admitted alive to the hospital, and excluded those who were transferred or died in the ED. We used linear regressions with survey weighting to compare differences in mean healthcare charges by firearm injury intent, with and without adjustment for ED disposition. RESULTS/ ANTICIPATED RESULTS: Among 12,469 cases in the weighted sample, mean age was 16.5 years, a majority were male (88.2\%) and Medicaid-insured (57.8\%), and 64\% were discharged from the ED and $36 \%$ admitted. Injuries were $49.0 \%$ unintentional, $45.1 \%$ assaultrelated, and $1.8 \%$ self-inflicted. Compared to children with self-inflicted injuries (charges $\$ 115,224$ ), children with assault-related injuries (charges \$55,052; $\mathrm{p}<0.007$ ) and unintentional injuries (charges $\$ 38,643$; $\mathrm{p}<0.001)$ had lower mean charges per visit. Differences in charges were no longer significant after adjusting for ED disposition, as $85.8 \%$ of self-inflicted injuries were admitted, compared to $46.5 \%$ of assault-related and $24.3 \%$ of unintentional injuries. DISCUSSION/ SIGNIFICANCE OF IMPACT: Although the majority of pediatric firearm-related injuries resulting in emergency department care are unintentional or assault-related, self-inflicted injuries result in greater per visit hospital charges, attributable to higher hospitalization rates, and likely due to more severe injuries.

4164

\section{Body mass index, not chemotherapy is the major predictor of insulin resistance in patients with hematological malignancies}

Melis Sahinoz ${ }^{1}$, Brian Engelhardt ${ }^{1}$, Dae Kwang Jung ${ }^{1}$, James Matthew Luther ${ }^{1}$, and Talat Alp Ikizler ${ }^{1}$

${ }^{1}$ Vanderbilt University Medical Center

OBJECTIVES/GOALS: Cancer survivors are at increased risk for type 2 diabetes mellitus.It is not clear if diabetes susceptibility is due to shared risk factors for cancer and diabetes, such as obesity, or if it is directly related to cancer and its treatment. We investigated the association between malignancy and insulin resistance, a major risk factor for diabetes. METHODS/STUDY POPULATION: 20 adult patients with treated hematological malignancies and 21 controls without cancer were included in the study. Individuals with pre-existing diabetes were excluded. All patients underwent a 2-hour 75-gram oral glucose tolerance test (OGTT). Hyperinsulinemiceuglycemic clamps were performed to measure the steady-state glucose infusion rate (M-value) as an indicator of whole-body glucose utilization during insulin stimulation. Insulin sensitivity index was calculated by dividing M-value over the steady-state plasma insulin (M/I). RESULTS/ANTICIPATED RESULTS: Fasting or postprandial plasma glucose levels during the OGTT did not differ significantly between malignancy patients and controls (Table 2). Difference in the insulin-stimulated glucose utilization (M-value) was not statistically significant among cancer patients and controls (median, 7.2 [IQR, 6.2-10.4] vs. 7.3 [IQR, 5.5-8.9] $\mathrm{mg} / \mathrm{kg} / \mathrm{min}$; $\mathrm{P}=0.261) . \mathrm{M} / \mathrm{I}$ index was significantly higher in malignancy patients compared to controls (median, $42.4 \mathrm{mg} / \mathrm{kg} / \mathrm{min} /(\mu \mathrm{U} / \mathrm{ml})$ [IQR, 33.967.2 ] vs. $23.4 \mathrm{mg} / \mathrm{kg} / \mathrm{min} /(\mu \mathrm{U} / \mathrm{ml})$ [IQR, 12.9-29.2], $\mathrm{P}<0.001)$, however insulin clearance was also lower in the controls. In multivariate analysis, only BMI was significantly associated with $\mathrm{M}$-value $(\beta=-0.2(95 \% \mathrm{CI}-0.4,-0.1), \mathrm{P}=0.004)$, and $\mathrm{M} / \mathrm{I}(\beta=-2(95 \%$ CI $-3.4,-0.5), \quad \mathrm{P}=0.009)$. DISCUSSION/SIGNIFICANCE OF IMPACT: Our data suggest that the major contributor to diabetes development after diagnosis of cancer in adults is obesity-induced insulin resistance, not malignancy related factors. These findings emphasize the importance of obesity management in long-term survival of cancer patients, as diabetes is a risk factor for mortality in this patient population. 